Phot oel ect r on Spect roscopi c St udy on Phot o- I nduced Phase Transi ti on of Spi n- Crossover Compl ex

\begin{tabular}{|l|l|}
\hline 著者 & $\begin{array}{l}\text { KANADA M TAKAHASH } \mathrm{K}, \text { DOI Y, FUKUI K, } \\
\text { TAYAGAK T, TANAKA K }\end{array}$ \\
\hline $\begin{array}{l}\text { j our nal or } \\
\text { publ i cat } \mathrm{i} \text { on } \mathrm{t} \text { i t l e }\end{array}$ & Phase Transi ti ons \\
\hline vol une & 75 \\
\hline number & $7-8$ \\
\hline page range & $847-853$ \\
\hline year & $2002-01$ \\
\hline URL & ht t p: //hdl . handl e. net /10098/1585 \\
\hline
\end{tabular}




\title{
PHOTOELECTRON SPECTROSCOPIC STUDY ON PHOTO-INDUCLD PHASE TRANSITION OF SPIN-CROSSOVER COMPLEX
}

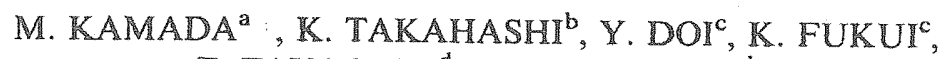 \\ T. TAYAGAKI ${ }^{\mathrm{d}}$ and $\mathrm{K}$. TANAKA \\ a Synchrotron Light Application Center, Saga University, Monjo 1, Saga 840-8502, Japan; \\ ${ }^{\mathrm{b}}$ UVSOR Facility, Institute for Molecular Science, Okazaki 444-8585, Japan, \\ "Faculty of Engineering, Fukui University, Fukui 910-8507, Japan; \\ dDeparment of Physics, Graduate School of Science, Kyoto University, Kyoto 606-8502, Japan
}

\begin{abstract}
Valence band structures in various phases of an organometal spin-crossover complex $\left[\mathrm{Fe}\left(2-\mathrm{pic}_{3}\right]_{\mathrm{Cl}} \cdot \mathrm{EtOH}\right.$ have been measured by means of photoelectron spectroscopy based on the combination of synchrotron radiation and laser light. The valence band structure showed the remarkable change due to the photo-induced (PI) phase transition (PIPT) at low-temperatures (LTs) as well as the thermally induced phase transition (TIPT), but the structure of the PI phase was very different from that of the high-temperature (HT) phase. The DV-X $\alpha$ calculation based on the $\left[\mathrm{Fe}-\left(\mathrm{NH}_{3}\right)_{6}\right]$ octahedron was also carried out for comparison, indicating that the electronic structure originating from $\mathrm{Fe}$ and $\mathrm{N}$ ions is closely related to the PIPT.
\end{abstract}

Keywords: Photo-induced phase transition; Spin-crossover complex; $\left[\mathrm{Fe}(2-\text { pic) })_{3}\right] \mathrm{Cl}_{2} \cdot \mathrm{EtOH}$; Photoelectron spectroscopy; Synchrotron radiation

\section{INTTRODUCTION}

Photo-induced (PI) phenomena have been attracting much interest (Nasu, 1996), because the microscopic electronic transition by photo-excitation can create macroscopic phase transition. Up to now, PI phenomena have been reported in several typical materials such as organic compounds (Koshihara et al., 2000), semiconductors (Koshihara et al., 1997), and superconductors (Ishikawa et al., 1999). An organometal spin-crossover complex $\left[\mathrm{Fe}(2-\text { pic })_{3}\right] \mathrm{Cl}_{2} \cdot \mathrm{EtOH}$ (2-pic=2-aminomethyl-pyridine) (hereafter abbreviated as Fe-pic) is one of the typical materials showing photo-induced phase transition (PIPT). This material also shows the thermally induced phase transition (TIPT) at critical temperatures $\left(T_{c}\right)$ of 114 and $121 \mathbb{K}$ (Gutlich et al., 1994). The high- and low-temperature (HT and $\mathrm{LT}$ ) phases correspond to the high-spin phase with $S=2$ and the low-spin phase with $S=0$, respectively. It shows the PIPT, 
when it is excited by green light at LTs. The chromatic and magnetic properties change drastically in the PIPT.

The PIPT has been investigated in typical materials with optical and/or magnetic methods (Decurtins et al., 1985; Hauser, 1991; Ogawa et al., 2000). However, the PIPT is not clearly understood yet, requiring systematic investigations with other methods. There is no experimental data about the electronic structures in the wide energy range, although the electronic structure is very important to understand PIPT. The purpose of the present study is to investigate the electronic structures in wide energy range for various phases of the Fe-pic crystal and also to know the relation between PIPT and electronic states. The valence-band photoelectron spectra of an Fe-pic crystal have been measured by means of the combination of synchrotron radiation and laser light. Moreover, the energy-band calculation has been carried out with the DV-Xa method based on the $\left[\mathrm{Fe}-\left(\mathrm{NH}_{3}\right)_{6}\right]$ cluster. The present results indicate that the photo-induced (PI) phase is very different from HT and LT phases. It is also indicated that the asymmetric distortion in the photo-excited states may play an important role in the PIPT of this material.

\section{CEPPDERIMENTAL}

Experiments have been carried out at the solid/surface photoelectron-spectroscopy beam-line in the UVSOR facility of Institute for Molecular Science, Okazaki. The ESCA system (FISONS Instruments, ESCALAB 220i-XL) was used to observe the photoelectron spectra. A hemispherical electron-energy analyzer was utilized in the experiments. Synchrotron radiation from the $750 \mathrm{MeV}$ electron storage-ring was monochromatized for ultraviolet photoelectron spectroscopy (UPS) with a planegrating monochromator. The overall energy resolution was about $0.3 \mathrm{eV}$. The $\mathrm{X}$-ray photoelectron spectroscopy (XPS) was also measured with $\mathrm{Mg}-\mathrm{K} \alpha$ lines from an X-ray tube for comparison.

Powders of Fe-pic were synthesized in Kyoto University without an oxygen atmosphere. Fe-pic single crystals were also grown from the solution. The typical size of the crystals was about $1 \times 1 \times 0.3 \mathrm{~mm}^{3}$. The sample was attached on the sample holder of a flow-type helium cryostat with a carbon adhesive tape. No paste was used to fix the sample, since the paste materials may react with the Fe-pic sample. The sample was then scraped with a knife-edge in the preparation chamber of less than $5 \times 10^{-8} \mathrm{~Pa}$. Any damage during cleaning the sample surface was not observed in the XPS spectra. The UPS spectra were measured at room temperature (RT), and then the sample was cooled down to about $40 \mathrm{~K}$. The TIPT was confirmed with observing the sample color by our eyes. The $\mathrm{Ar}^{+}$ion laser of about $9 \mathrm{~mW}$ in multi-line mode was introduced on the sample surface to cause the PIPT. The sample colors showed yellow and red at $\mathrm{RT}$ and $40 \mathrm{~K}$, respectively, and the laser excitation at $40 \mathrm{~K}$ changed the sample color to yellow.

\section{RESULTS}

Figures $1 \mathrm{a}, \mathrm{b}$ show the valence-band spectra of the Fe-pic single crystal at RT and $40 \mathrm{~K}$, respectively. The peaks a and $\mathrm{b}$ are observed around 11 and $15 \mathrm{eV}$, respectively at $R T$. At $40 \mathrm{~K}$, structures $A, B, C$, and $D$ are observed around $7,10,15$, and $17 \mathrm{eV}$, 


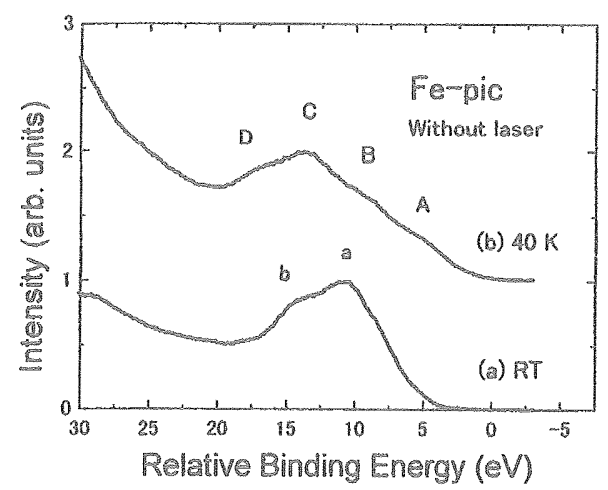

FIGURE 1 URS spectra of Fe-pic crystal at RT (a) and $40 \mathrm{~K}$ (b).

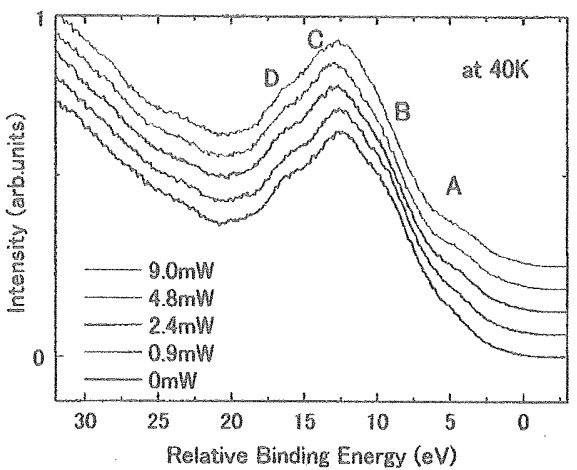

FGURE 2 Photo-induced change in the UPS spectra of Fepic crystal at 40 K under various laser intensities.

respectively. It is stressed that the valence-band structures are very different from each other between RT and $40 \mathrm{~K}$. Figure 2 shows the valence-band spectra under the laser excitation at $40 \mathrm{~K}$. The laser excitation at $R T$ did not cause any change in the photoelectron spectra, while the laser excitation at $40 \mathrm{~K}$ showed the remarkable change due to the PIPT as shown in the figure. It is obvious that the peak $\mathrm{A}$ around $5 \mathrm{eV}$ becomes intense and the shoulder $\mathbb{B}$ disappears under laser excitation.

Figure 3 shows the change in the intensity of the peak $A$, shoulder $B$, and the peak $C$ at $40 \mathrm{~K}$ as a function of the laser power. The peak $\mathrm{A}$ was increased by about $35 \%$ at the laser power of $9 \mathrm{~mW}$. The intensity of the shoulder $B$ was decreased by about $17 \%$ at the laser power of $9 \mathrm{~mW}$ and therefore the absolute value of the decrease is plotted in the figure. The change in the peak $C$ was so small as to be shown in the expanded scale. The figure shows that the dependence of the increment or decrement in the valence-band intensity on the laser power is not simple. The dependence is not linear in the whole range of the laser power, but shows the saturation behavior at higher laser power. The threshold was not clearly observed in the present experiment, but this does not mean that there is no threshold intensity for PIPT. Since the present experiment is not good enough to observe the threshold, detailed experiments are required to confirm it. The present result shown in Fig. 3 indicates that the PIPT affects the whole range of the valence states. 


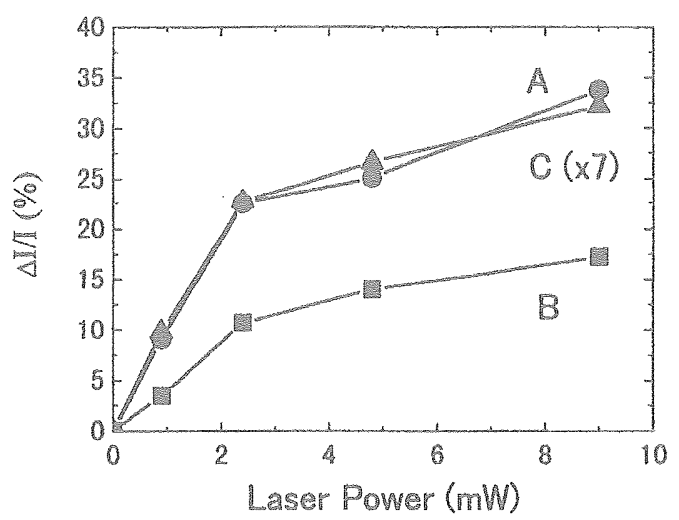

FIGURE 3 Photo-induced change in the UPS spectra of Fe-pic crystal as a function of laser power.

The XPS spectra have been obtained for comparison. The Cl-2p spectrum showed the energy shift in the TIPT, due to the thermal expansion of the crystal lattice, but did not show any change in the PIPT. The $\mathrm{O}-1 \mathrm{~s}$ and $\mathrm{C}-1 \mathrm{~s}$ peaks showed the same energy shift as that of the $\mathrm{C}-2 \mathrm{p}$ peak. The $\mathrm{N}-1 \mathrm{~s}$ peak was shifted to higher binding-energy side both for TIPT and PIPT.

\section{DISCUSSION}

It is obvious from Figs. 1 and 2 that the valence-band spectra in the LT, HT, and PI phases are different from one another, although the color of the sample is red, yellow, and yellow for LT, HT, and II phases, respectively. The XPS result supports that the electronic structures of the $\mathbb{L T}, \mathrm{HT}$, and PI phases are different from one another. Recently, the Raman spectroscopy has been carried out on the Fe-pic (Tayagaki and Tanaka, 2001), which showed many low-frequency vibrational modes were observed only in the PI phase, but not in the HT phase. Therefore, it is concluded that the PI phase is not the same as the HT phase, although the sample color is similar in both phases.

According to the photoelectron spectra of Fe-compounds, the $3 d$ electrons are located around the top of the valence-band (Shimada et al., 1998). The present UPS spectra distribute in the wide energy range, indicating the contribution from nitrogen ions surrounding the $\mathrm{Fe}$ ion. As shown in Fig. 2, the peak $\mathrm{A}$ is clearly observed around the top of the valence-band in the PI phase, while the peak is not clearly appreciable in the HT phase. Therefore, the present results strongly indicate that the $\mathrm{Fe}-\mathrm{N}$ bond lengths may be changed by the photo-excitation and then the distributions of the $3 d$ electrons of $\mathrm{Fe}$ ions and the $2 \mathrm{~s} / 2 \mathrm{p}$ electrons of $\mathrm{N}$ ions change dramatically their distribution in the PIPT.

In order to understand the PIPT of an Fe-pic crystal in more details, the electronic structures were calculated with a DV-Xa method (Adachi et al., 1978). The crystal structure of $F$ e-pic is $P 2_{1} / c, Z=4$, over the entire temperature range between $90 \mathrm{~K}$ and $\mathbb{R T}$ (Mikami et al., 1980). The $\mathrm{Fe}-\mathrm{N}$ bond lengths show remarkable changes according to the electron configuration of the two spin states in the TIPT. The average bond lengths are 2.013 and $2.195 \mathrm{~A}$ in the $\mathrm{LT}$ and HT phases, respectively. All the amino $\mathrm{N}$ atoms of the complex are hydrogen bonded to $\mathrm{Cl}^{-}$forming a two-dimensional 
network parallel to the (100) plane. Solvate ethanol molecules, hydrogen bonded to $\mathrm{Cl}^{-}$, exhibit orientational disorder in the HT phase, but a regular arangement in the LT phase. Since the Fe-pic crystal has complicated crystal structures with many elements as mentioned above, the calculation was carried out on an $\left[\mathrm{Fe}-\left(\mathrm{NH}_{3}\right)_{6}\right]$ octahedron in a frame of the Fe-pic crystal, where a picoryleamino molecule was replaced by $\mathrm{NH}_{3}$, with neglecting EtOH and $\mathrm{Cl}$ atoms for simplicity.

Figure 4 shows the comparison of the calculated density of states (DOSs) with the observed UPS spectra. The valence state of the $L T$ phase consisis of three nonbonding states $\left(t_{2 \mathrm{~g}}\right)$ originating from the $\mathrm{Fe}-3 \mathrm{~d}$ orbital and six bonding states $\left(e_{\mathrm{g}}, a_{\mathrm{ig}}\right.$, and $\left.t_{1 \mathrm{u}}\right)$ from Fe-3d, $-4 s,-4 p$ and $N-2 s,-2 p$ orbitals in the case of $\mathrm{Fe}^{2+}$ configuration. The nonbonding and bonding states in the $\mathrm{Fe}^{3+}$ configuration also contribute the valence-band spectra. The simple total sum of the DOSs in the $\mathrm{Fe}^{2+}$ and $\mathrm{Fe}^{3+}$ configurations is shown in the figure. The spectral features in the $0-7,7-12$, and $12-16 \mathrm{eV}$ range of the LT phase may be attributed to the $t_{2 \mathrm{~g}}, e_{\mathrm{g}}+a_{1 \mathrm{~g},}$ and $t_{13}$ states, respectively, but any prominent peak corresponding to the $\tau_{2 g}$ state in the $\mathrm{Fe}^{2-t}$ configuration is not clearly observed around the top of the valence-band spectrum. The spectral feature around $10 \mathrm{eV}$ and the lower energy part in the HT phase may be attributed to the $t_{\mathrm{iu}}+a_{\mathrm{gg}}$ and $e_{\mathrm{g}}+t_{2 \mathrm{~g}}$ states, respectively, but any prominent peak corresponding.
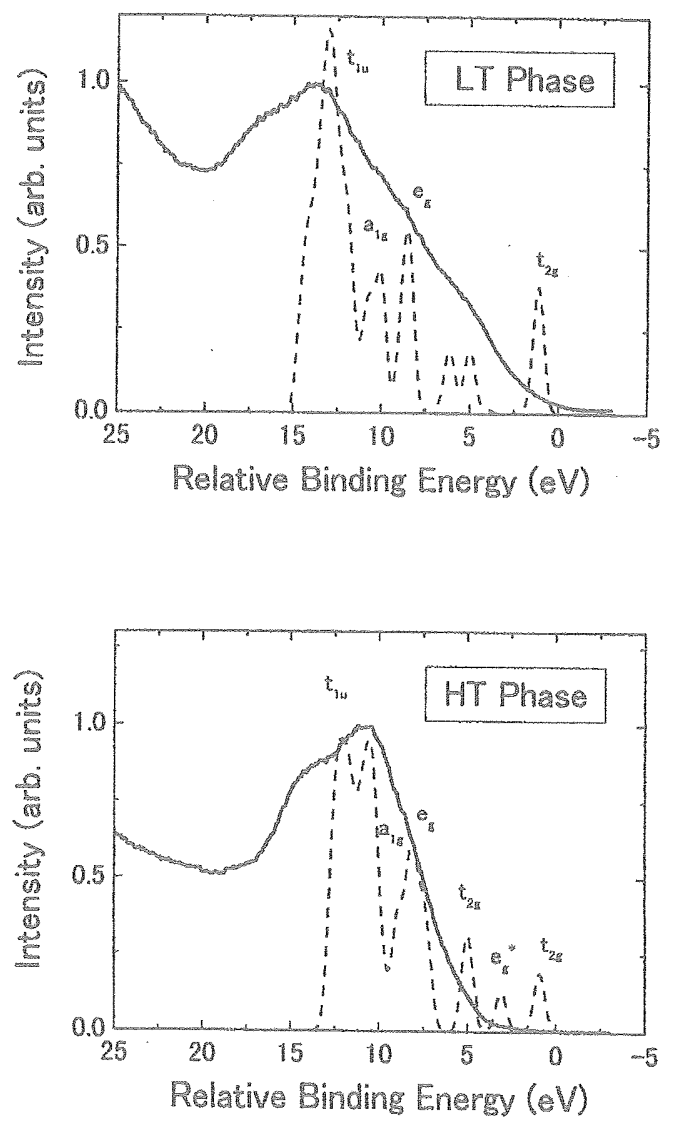

FGURE 4 Comparison between the calculation and UPS spectra for HT and LT phases. 
to the $t_{2 \mathrm{~g}}+e_{\mathrm{g}}^{*}$ state in the $\mathrm{Fe}^{2+}$ configuration is not clearly observed around the top of the valence-band spectrum. The present calculation is too simple to include the configurational interaction between the final states and also the contribution from other constitute elements is not considered in the calculation, but it gives the fairly good agreement with the photoelectron spectra. The discrepancy between the calculation and the spectra around the top of the valence-band may be due to the simplicity of the calculation and/or the small contribution from the $\mathrm{Fe}^{2+}$ configuration.

The crystal structure of the PI phase is not clear at the present stage, since the PI phase can be observed only by the photo-excitation, but not in the stational condition. The recent Raman spectroscopy carried out on the Fe-pic (Tayagaki and Tanaka, 2001) showed many low-frequency vibrational modes, indicating the symmetry lowering in the PI phase. The asymmetric distortion cannot be directly induced by the thermal energy, since the TIPT occurs between the LT and HT phases, both of which belong to the same symmetry. This strongly suggests the asymmetric distortion in the excited state $\left({ }^{1} T_{1}\right.$ or $\left.{ }^{1} T_{2}\right)$, but not in the ground state. Therefore, it was assumed in the present calculation that the $E_{\mathrm{g}}$-distortion due to the Jahn-Teller instability, which corresponds to the elongation of the Fe-octahedron along the $z$-axis, may play an important role in the PI phase. It was found from the calculation that the $E_{\mathrm{g}}$-distortion more than $3 \%$ in the LT state can cause the phase transition into another spin state. Another distortion $T_{2 \mathrm{~g}}$ (not shown here) had less efficiency to cause the PIPT. Since the PI phase is known to have the magnetism (Ogawa et al., 2000), the electronic structures for up-spin and down-spin states are expected to be shifted from each other. Namely, the top of the up-spin states may be the anti-bonding state $\left(e_{\mathrm{g}}^{*}\right)$ originating from Fe-3d and $\mathrm{N}-2 \mathrm{~s},-2 \mathrm{p}$ orbitals, while that of the down-spin states is the nonbonding states $\left(t_{2 \mathrm{~g}}\right)$. It was found that the electronic structure originating from the $\mathrm{Fe}^{3+}$ configuration was not affected by the $E_{\mathrm{g}}$ - and $T_{2 \mathrm{~g}}$-distortions, while that from $\mathrm{Fe}^{2+}$ configuration was drastically changed by the distortions.

As shown in Fig. 3 , the peak $A$ is remarkably enhanced and also the shoulder $\mathbb{B}$ is decreased in the PIPT. This experimental result can be qualitatively explained with the calculation mentioned above. As shown in Figs. 4 and 5 , the $e_{\mathrm{g}}^{*}+t_{2 \mathrm{~g}}$ states corresponding to the peak $\mathrm{A}$ is enhanced and the $e_{\mathrm{g}}$ state corresponding to the shoulder $\mathrm{B}$ is shifted in the PI phase. Since $e_{\mathrm{g}}$ and $e_{\mathrm{g}}^{*}$ states are bonding and anti-bonding states between Fe-3d and N-2s/2p-orbitals, respectively, it is therefore proposed that the PIPT is trigerred by the change in the $\mathrm{Fe}-\mathrm{N}$ bond due to the symmetry lowering. The asymmetric distortion in the excited states is very important in the PIPT. The $J a h n-T e l l e r$ type distortion in the $\mathrm{Fe}^{2+}$ configuration is one of the candidates to cause the PIPT, but further investigations are required to confirm it.

Finally, it is reported that the increment of the peak A due to the PIPT showed the dramatic increase around $110 \mathrm{~K}$, when the sample was heated up from the LT to HT phases. This PI increment of the peak A decreases instantly after the photoexcitation is stopped. This is very interesting and will be reported in near future.

In conclusion, the electronic structures of the Fe-pic crystal has been studied in $L T$, HT, and PI phases by photoelectron spectroscopy based on the combinational use of the synchrotron radiation and laser light. The valence-band structure showed the remarkable change due to the PIPT at LT as well as the TIPT, but the structure of the PI phase was very different from that of the HT phase. The DV-Xot calculation based on the $\left[\mathrm{Fe}-\left(\mathrm{NH}_{3}\right)_{6}\right]$ octahedron was also carried out for comparison. The present 


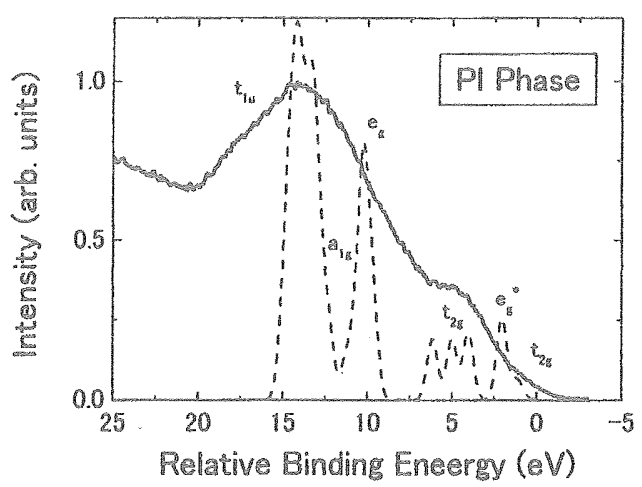

FIGURE 5 Comparison between the calculation and UPS spectrum for PI phase.

results indicate that the PI phase is very different from LT and HT phases and also that the PIPT may be caused by the change in the electronic structures originating from the Fe-N bond due to the symmetry lowering.

\section{A}

The authors express their sincere thanks to Dr. Y. Haruyama of LASTI, Himeji Institute of Technology and Prof. T. Kinoshita of ISSP, Tokyo University for their kindness to use photoelectron spectrometer. This work was partially supported by a Grant-in-Aid for Scientific Research on Priority Area, "Photo-induced Phase Transition and Their Dynamics" from the Ministry of Education, Science, Sports, and Culture, Japan.

\section{References}

Adachi, A., Tsukada, M. and Satoko, C. (1978). J. Phys. Soc. Jpn., 495, 875.

Decurtins, S., Gutlich, P., Hasselbach, K.M., Hauser, A. and Spiering, H. (1985). Inorg. Chem., 2A, 2174.

Gulich, P. Hauser, A. and Spiering, H. (1994). Angew. Chem. Int. Ed. Engl., 33, 2024.

Hauser, A. (1991). J. Chem. Phys., 98, 2741.

Ishikawa, H., Ookura, K. and Tokura, Y. (1999). Phys. Rev., 啙59, 8367.

Koshihara, S (2000). J. Luminescence, $97 / 969,77$.

Koshihara, S., Oiwa, A., Hirasawa, M., Katsumoto, S., Iye, Y., Urano, C., Takagi, H. and Munekata, H. (1997). Phys. Rev. Lett., 78, 4617.

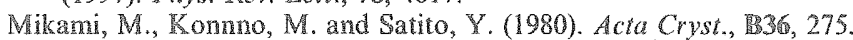

Nasu, K. (1996). Relaxations of Excited States and Pholo-Induced Structural Phase Transitions. Springer, Berlin, Heidelberg, New York.

Ogawa, Y., Koshihara, S., Koshino, K., Ogawa, T., Urano, C. and Takagi, H. (2000). Phys. Rev. Lett., 84, 3181.

Shimada, K., Mizokawa, T., Mamiya, K., Saitoh, T., Fujimori, A., Ono, K., Kakizaki, A., Ishi, T., Shirai, M. and Kamimura, T. (1998). Phys. Rey.,

Tayagaki, T. and Tanaka, K. (2001). Phys. Rev. Letr., $866,2886$. 